\title{
Aspectos estructurales del comercio fronterizo entre México y Estados Unidos
}

\author{
Bernardo González-Aréchiga \\ Centro de Estudios Fronterizos del Norte de México
}

\begin{abstract}
Resumen
El objetivo de esta presentación es discutir algunos aspectos estructurales de la frontera y someter a discusión la estrategia de investigación del Departamento de Estudios Económicos de CEFNOMEX. Aquí se explica cómo la frontera norte cuenta con una estructura comercial dual con patrones variables de consumo de servicios en Estados Unidos, tales como los educacionales, médicos, asistenciales, etc., que han afectado a la creación de una infraestructura local, y que la zona fronteriza del norte de México exhibe importantes distorsiones estructurales que deben estudiarse para entender sus funcionamiento económico. Es importante mejorar la base informativa con recolección directa. La investigación económica de la región requiere tanto del estudio de sus instituciones, como del monitoreo constante y cuidadoso de sus signos vitales. Una estrategia adecuada de investigación debe combinar ambos y aprovechar las complementariedades de los dos tipos de estudio.
\end{abstract}

Palabras clave: comercio fronterizo, comercio informal, frontera, estructura comercial dual, patrones de consumo.

\begin{abstract}
The objective of this presentation is to discuss on some of the structural aspects of the border and to bring into the research strategy of the Department of Economic Researches at CEFNOMEX. This document explains that the Northern border has a dual commercial structure with variable service business patterns of purchases in the United States, such as educational, medical, assistance, etc., that have affected the formation of local infrastructure. Also to explain that the border zone of the North of Mexico shows considerable structure distortions that should be studied to understand its economical performance. It is important to improve the informative sources by direct collection. Economical researches of the region require both the study of the institutions, and also to perform a steady and careful follow up of its vital signs. A proper research strategy should combine both and take advantage of the complementarities of the two forms of study.
\end{abstract}

Keyword: border trade, informal business, border, dual business structure, purchase patterns. 


\title{
ASPECTOS ESTRUCTURALES DEL COMERCIO FRONTERIZO ENTRE MEXICO Y ESTADOS UNIDOS*
}

\author{
Por \\ Bernardo González-Aréchiga
}

\section{INTRODUCCION}

La región fronteriza de México con Estados Unidos tiene estructuras económicas propias que responden a las condiciones legales, y a los comportamientos económicos binacionales. El desarrollo fronterizo conlleva el desarrollo de instrumentos económicos e instituciones propias. El estudio de la región tiene que considerar la formación de las estructuras autóctonas, sus efectos en el funcionamiento de la maquinaria económica y su impacto en las alternativas de política económica.

El objetivo de esta presentación es discutir algunos aspectos estructurales de la frontera y someter a discusión la estrategia de investigación del Departamento de Estudios Económicos de CEFNOMEX.

\section{La estructura comercial}

La región fronteriza debe entenderse a la luz de los procesos de rápida migración y acelerada creación de infraestructura. La región entera está marcada por la gravitación que ejerce Estados Unidos sobre voblación y recursos, y los comportamientos propios que ofrece un territorio todavía escasamente. explotado. El balance entre gravitación externa y recursos propios estuvo dictado en periodos muy largos por la sobrevaluación, a veces grave, del peso mexicano en relación con el dólar estadounidense. La población incorporada a la región rápidamente aprendió a vivir en el entorno binacional en el que se subsidiaban todos los artículos externos, por la sobrevaluación del peso. Los hábitos de la población migrante, originalmente orientados hacia productos nacionales, se fueron moldeando al nuevo ambiente y hacia los productos estadounidenses.

La infraestructura también creció distorsionada por el subsidio cambiario. Los establecimientos comerciales que surgieron en el periodo de sobrevaluación estuvieron dirigidos a satisfacer las necesidades de una población

\footnotetext{
*Ponencia presentada para el “Colloquium of Recento Quantitative Research on Mexico's Economy and Society". University of California, Los Angeles, marzo 4 y 5, 1985.

El autor es investigador del Centro de Estudios Fronterizos del Norte de México (CEFNOMEX) y jefe del Departamento de Estudios Económicos.
} 
con hábitos binacionales. La distribución y el comercio se especializaron en bienes no importables (por imposibilidad física o legal) y en la prestación de servicios que se consumen, por su naturaleza, en el lugar de residencia. Las demandas intermedias del comercio también se adquirirían en el exterior, dificultando la formación de canales de abasto y/o la producción regional de los mismos.

La transportación de mercancías nacionales hacia la frontera y la formación de redes comerciales fueron igualmente inhibidos por las condiciones cambiarias. El resultado es que la frontera se desarrolló como un mercado independiente, envidiable para los productores nacionales pero poco confiable. En este ambiente se dificultó el establecimiento de lazos comerciales durables entre la frontera y el centro del país; esto ha dañado tanto a consumidores fronterizos como a abastecedores nacionales, ya que la permanencia de las relaciones comerciales es una precondición para el abasto confiable y la programación adecuada de la producción.

Una prueba de la distorsión estructural causada por la sobrevaluación del peso es la tendencia reciente a exportar capacidad empresarial a Estados Unidos; el hecho de que las redes de comercialización fronteriza estuvieran conectadas hacia el extranjero permitió a los comerciantes establecer lazos permanentes con abastecedores externos. La permanencia de esta relación representa confianza mutua y el otorgamiento de créditos, descuentos por pronto pago de los abastecedores que aumentan la rentabilidad comercial. Esto es particularmente importante en negocios en los que la rotación de inventarios es baja. Con la recesión de 1982 y la rápida devaluación del peso, algunos comerciantes han decidido abrir establecimientos en Estados Unidos. Esta operación les permite capitalizar las relaciones comerciales de muchos años, atacando el mercado estadounidense y el de mexicanos que compran en el exterior. En primera instancia esto parecería comprender, simplemente, la ampliación de las operaciones comerciales y la diversificación de mercados; sin embargo, el proceso tiene una segunda faceta porque permite la transferencia de capital de trabajo, de un lado a otro de la frontera. Así, logran de facto crear toda una gama de instrumentos para la reasignación internacional de capital de trabajo y capacidad empresarial. Este proceso puede llevar a la descapitalización de algunos comercios fronterizos y a la fuga de capitales, o bien a una simple diversificación sin consecuencias negativas para la región.

Otro aspecto de la frontera es la constante alteración de las reglas del juego binacional: los códigos aduanero: los permisos de importación, los planes de industrialización fronteriza y el resto del aparato legal cambian frecuentemente; también cambia el celo con el que, efectivamente, se vigila su cumplimiento. El mismo tráfico de personas está sujeto al capricho de quie- 
nes toman las decisiones y de muchas dependencias públicas en ambos países. Este elemento ha marcado la infraestructura de la región en todos sus niveles.

\section{Estructura comercial informal}

La población fronteriza mexicana ha dado respuesta a las reglas binacionales permanentes y a su cambio de una forma espontánea,creando a través de los años, una estructura informal muy poderosa. Esta estructura incluye los swap meets ${ }^{1}$, las "segundas", las redes de reciclaje de productos usados y basura de Estados Unidos, y las redes familiares de abasto. Todas estas instituciones demuestran fehacientemente la incapacidad del sector comercial formal para satisfacer las necesidades de los grupos más pobres. La respuesta del sector informal a productos empaquetados son las redes de reciclaje, que adquieren (a veces como desechos) productos defectuosos o usados en Estados Unidos, los transforman (limpian y arreglan) y los venden en tiendas de segunda o swap meets. Estas instituciones podrían ser consideradas como variantes de los mercados dominicales del centro de México, o como adaptaciones de Estados Unidos; sin embargo, su carácter es único por ser efectivamente binacionales y por ser una alternativa para el sector formal.

Las redes familiares de consumo son también de gran importancia, ya que permiten a grupos amplios realizar compras directas en Estados Unidos, aun cuando sólo un pequeño número de ellos tiene acceso legal a aquel país. Familiares y amigos coordinan sus compras y comparten información sobre precios, logrando mejorar su conocimiento del mercado externo. Este mecanismo amplía enormemente el número de compradores potenciales y permite diseminar experiencias indirectas de participación en los mercados internacionales.

La población ha creado un mercado "paralelo" para los pobres: de hecho, no se pueden entender los patrones comerciales de los fronterizos ni evaluar su nivel de bienestar, sin considerar las estructuras generadas por el sector informal y los productos que maneja. Bien podría ser que el supuesto alto nivel de vida de los fronterizos (especialmente los bajacalifornianos) no fuera sino un espejismo estadístico que reflejara no altos niveles de vida, sino la modernización binacional de la pobreza.

$1 \mathrm{El}$ swap meet es una forma de mercado que concentra a un gran número de pequeños vendedores, en un espacio normalmente abierto. Tanto los vendedores, como los compradores pagan por participar. Los primeros rentan a los organizadores o al dueño del baldío el espacio que ocupan; los compradores pagan una módica cuota por entrar. Algunos swap meets son permanentes y otros se abren solamente los domingos y días festivos. Los principales productos que se venden en los swap meets son ropa nueva y usada, bienes duraderos y aparatos electrónicos usados, alfombras, partes para automóviles, refacciones electrónicas, herramientas de segunda mano y cassetes con grabaciones piratas. 
Tampoco puede entenderse cabalmente la falta de competitividad de los productos de manufactura nacional en la frontera, sin considerar los aspectos estructurales descritos. La competitividad no es una cualidad intrínseca de las mercancías, sino una cualidad global de los sistemas productivo y distributivo. Afirmar que los productos mexicanos no compiten con productos estadounidenses en la frontera, equivale a escatimar la importancia del sector comercio; la realidad es que el sistema comercial fronterizo con bienes mexicanos y extranjeros, compite con el sistema comercial estadunidense, que vende tanto productos locales como importados de todo el mundo. Las limitaciones estructurales de los sectores comerciales restan competitividad a los productos nacionales, pues éstos reciben todas las ineficiencias de transportación y mercado.

La escasa aceptación de los bienes mexicanos en la frontera no es indicador confiable de la competitividad de la maquinaria productiva nacional en el exterior; pero sí es un buen indicador de la ineficiencia de su sistema de producción y/o distribución. Hay, sin embargo, algunos tipos de bienes en los que el rechazo a la producción nacional revela una falta real de competitividad a nivel productivo, y no se puede atribuir a una formidable ineficiencia comercial. Entran en esta categoría ropa, calzado y artículos plásticos para el hogar de manufactura coreana, vietnamita, de Taiwán y otros países asiáticos. Estados Unidos es un gigantesco comprador de estos productos, los cuales se filtran, especialmente a través de California, hacia México. Lo interesante de este fenómeno, es que muestra que la maquinaria productiva asiática está más capacitada que la local para satisfacer las necesidades de la población fronteriza en ciertos productos de consumo popular. Esta conjetura es importante porque lleva a cuestionar la orientación y la capacidad innovadora de la industria mexicana.

\section{Población y estructura económica}

La afluencia de población a la frontera ha sido uno de los determinantes de la rápida creación de infraestructura y de las modalidades que ha tomado. Esta relación es obvia; pero conviene replantearse en términos de la participación de la población fronteriza en los mercados estadounidenses. Los rápidos cambios demográficos han afectado la demanda de servicios en una forma que no es típica de todas las regiones del país. La diferencia estriba en que existe en la frontera un "colchón" entre demanda y oferta de servicios; ese "colchón" es la contratación de servicios de Estados Unidos. El cambio demográfico modifica, estructuralmente, la posibilidad de contratar servicios en el exterior, y, por consiguiente, afecta en forma no lineal la necesidad de satisfacerlos localmente. Por ejemplo, que alguien pueda nacer en los hospitales de San Diego depende de las características migra- 
torias de los padres: las alternativas de ciudadanía, educación y empleo son sumamente importantes tanto individual como socialmente. Todo esto se modifica con la migración.

El rápido crecimiento de la población en la frontera ha cambiado fuertemente el panorama respecto de quién puede trabajar o estudiar en Estados Unidos, y quién puede comprar directamente o atenderse médicamente en aquel país. Estos cambios en los patrones de asociación o vinculación internacional han afectado fuertemente la formación de infraestructura de servicios en la región. El sector local de servicios se orienta a satisfacer las necesidades de aquellos grupos que no pueden satisfacerlas en el exterior. Con el tiempo, esta tendencia se ha solidificado y constituye parte del panorama estructural.

\section{La estructura financiera}

Así como los residentes fronterizos han desarrollado instituciones de comercialización, distribución y reciclaje consistentes con la dinámica binacional, también han creado toda una serie de instrumentos y prácticas financieras autóctonas. El mercado de bienes y servicios permite a la población satisfacer necesidades actuales. El mercado financiero les permite escoger cuándo hacerlo, es decir, facilita la transmisión de valor en el tiempo, adicionalmente, en un contexto binacional, les permite escoger en qué moneda hacerlo y con qué instrumentos financieros y bancarios. En la frontera hay un mundo de oportunidades financieras propicias para que inversionistas bien informados disfruten de las ventajas que ofrecen las instituciones de ambos países. El mayor obstáculo, fuente de gran creatividad, es que el número de instrumentos financieros que permiten la ágil transferencia de recursos de un país a otro es, por diseño, sumamente reducido. Los inversionistas han desarrollado mecanismos para lograrlo.

El mecanismo más obvio es el establecimiento de casas de cambio que aumentan la liquidez transfronteriza, escapando al racionamiento de dólares en el sistema bancario. Un segundo mecanismo es la facturación de contratos en dólares, aun en el caso de transacciones totalmente mexicanas, o la aceptación de cheques y otras formas de pago sin validez internacional legal. Existe un sinfín de métodos diseñados para aumentar la aceptación y la liquidez en México de instrumentos bancarios estadounidenses.

Es importante señalar que solamente una minoría de la población tiene acceso a esta flexibilidad financiera, y son, por supuesto, quienes ganan. El resto no tiene acceso a estas ventajas (excepto la posibilidad de tener dólares bajo el colchón), y están condenados a depender de los ins- 
trumentos bancarios y financieros nacionales que son inapropiados -a la luz de los comentarios anteriores- para los fronterizos. Así como se habla de una "banca paralela" a nivel nacional que incluye casas de bolsa, aseguradoras, etc.. se debe hablar de un sistema financiero "informal" en la frontera, pues es una de las estructuras económicas más importantes de la región.

\section{La investigación cuantitativa}

La investigación cuantitativa de la zona fronteriza del norte de México se enfrenta a un gran problema de falta de información estadística. Por un lado, se requiere la estimación de cuentas regionales desagregadas y cuentas microeconómicas de producción y consumo, necesarias para cualquier región. Por el otro, se necesita estimar los indicadores propios de una economía binacional, y medir sus aspectos estructurales. Las necesidades de información son enormes.

El Departamento de Estudios Económicos del CEFNOMEX, ha desarrollado una estrategia para la generación de indicadores económicos y modelos analíticos bajo el título: "Proyecto de Monitoreo Económico de la Frontera." 2 Este proyecto consiste en le seguimiento de un número seleccionado de variables y su procesamiento por medio de modelos económicos para la estimación de indicadores no observables o costos a observación. La base fundamental del proyecto es la recolección directa de información por medio del muestreo esatdístico.

La figura 1 resume el proceso de estimación que se aplica al caso del monitoreo. El punto de partida, representado por el círculo inferior izquierdo, es la captación muestral de información; el punto final, dado por el rectángulo de la derecha, es la estimación de los indicadores buscados. Las fases intermedias resumen, grosso modo, dos estrategias alternativas para llegar de la información muestral a los indicadores económicos. La alternativa descrita por el flujo superior consiste en la captación directa de información sobre fenómenos cercanamente relacionados con el fenómeno bajo estudio, y en su procesamiento para estimar los indicadores de interés. La transformación se hace por medio de definiciones contables -por ejemplo, en el caso de cuentas regionales- o de modelos explicativos previamente estimados. Esta técnica indirecta es utilizada porque frecuentemente es más barata que la observación directa, o porque las variables estudiadas son, simplemente, no observables. Por ejemplo, es virtualmente imposible medir la cantidad real de depósitos líquidos en bancos estadouni-

$\overline{2 \mathrm{El}}$ proyecto de monitores fue originalmente diseñado por Jorge A. Bustamante, Gustavo del Castillo y Kenneth Shellhammer, y está siendo revisado e implementado por Efraín Caro, José Luis Contreras, Gustavo del Castillo, Bernardo González Aréchiga, José Javier Robles y María Ramona Valdez. 
denses, la fuga de capitales o la magnitud del mercado negro. Aun en estos casos, es posible estimar estas transacciones con el uso de información indirecta y algunos supuestos de comportamiento.

La segunda alternativa, descrita en la parte inferior del diagrama, es la medición directa de indicadores, que si bien es más cara, también es más confiable. Es de esperarse que a medida que se estudie la frontera se dejará atrás la medición indirecta de indicadores observables y se perfeccionarán los modelos económicos. La técnica indirecta se seguirá utilizando mientras existan actividades económicas clandestinas o actividades legales estigmatizadas. Por el momento, la investigación regional requiere de la priorización de las necesidades de información y la selección de una estrategia viable para su obtención.

Las funciones prioritarias son el diseño e implementación de modelos regionales explicativos y la obtención de marcos de muestreo. Estos modelos son instrumentos para entender los aspectos estructurales de la frontera y diseñar la estrategia de monitoreo. La obtención de marcos confiables de muestreo sobre hogares, industrias, comercios y cruces fronterizos es sumamente importante, porque permite la rápida implementación de programas de recolección de información en las áreas claves de la economía.

FIGURA 1. Proceso logístico de estimación de indicadores económicos.

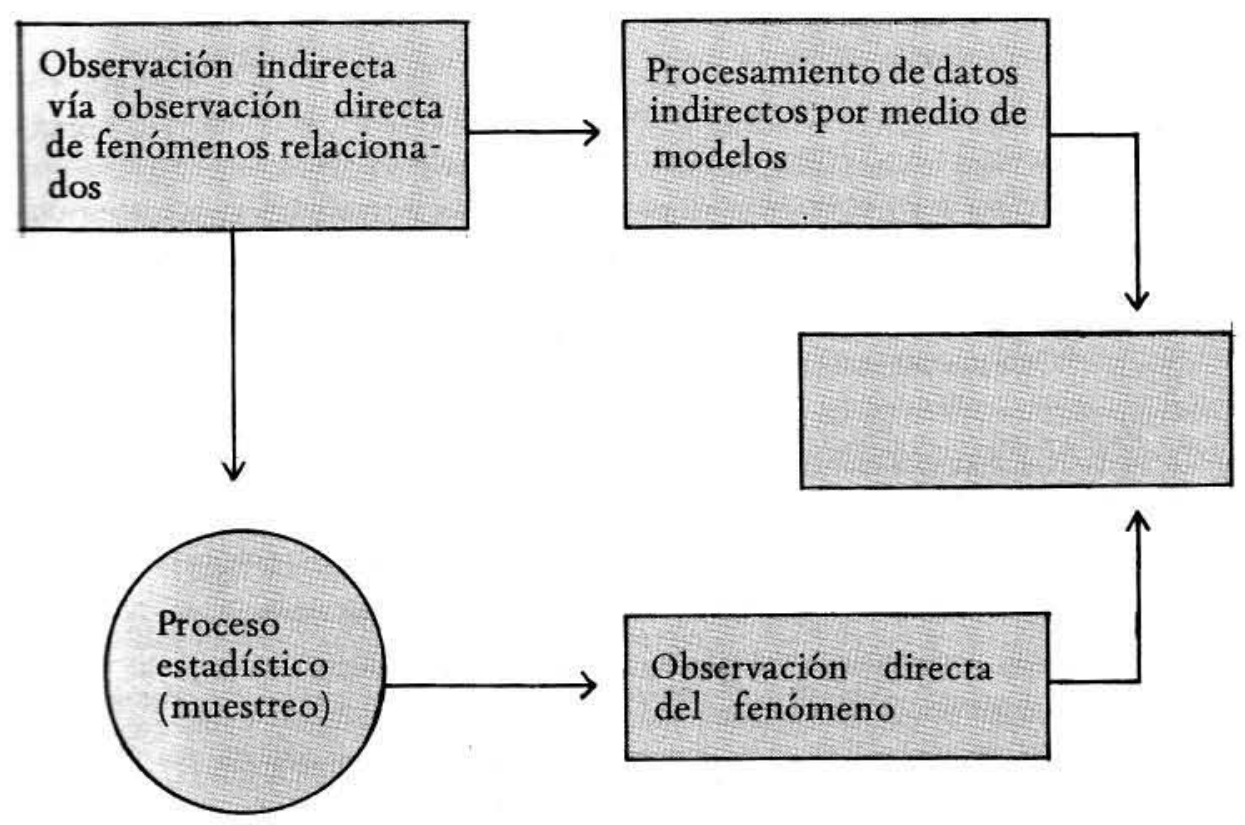




\section{CONCLUSIONES}

La zona fronteriza norte de México tiene una estructura comercial dual, con fuertes sectores formal e informal: un sector financiero informal complementario al sistema bancario nacional, y patrones variables de consumo de servicios (educacionales, médicos, asistenciales) en Estados Unidos, que han afectado a la creación de una infraestructura local. A esto han contribuido el tipo de cambio, la composición de la población, la influencia económica de Estados Unidos y las políticas que regulan la interacción binacional. El tipo de cambio es especialmente importante porque la sobrevaluación del peso, predominante en los últimos veinte años, ha impactado los patrones de consumo y la orientación productiva y comercial. Esto se ha materializado por medio de la acumulación de capital, la formación de hábitos de consumo y el establecimiento de lazos comerciales internacionales. La zona exhibe importantes distorsiones estructurales que deben estudiarse para entender su funcionamiento económico; la información estadística es escasa y dificulta la estimación de los indicadores básicos. Es importante mejorar la base informativa con recolección directa. La investigación económica de la región requiere tanto del estudio de sus instituciones, como del monitoreo constante y cuidadoso de sus signos vitales. Una estrategia adecuada de investigación debe combinar ambos y aprovechar las complementariedades de los dos tipos de estudio. 